\title{
Measuring the upset of CMOS and TTL due to HPM-signals
}

\author{
N. Esser and B. Smailus \\ ABB AG Corporate Research Center, PO Box 1140, 68520 Ladenburg, Germany
}

\begin{abstract}
To measure the performance of electronic components when stressed by High Power Microwave signals a setup was designed and tested which allows a well-defined voltage signal to enter the component during normal operation, and to discriminate its effect on the component.
\end{abstract}

The microwave signal is fed to the outside conductor of a coaxial cable and couples into the inner signal line connected to the device under test (DUT). The disturbing HF-signal is transferred almost independent from frequency to maintain the pulse shape in the time domain. The configuration designed to perform a TEM-coupling within a $50 \mathrm{Ohm}$ system prevents the secondary system from feeding back to the primary system and, due to the geometrical parameters chosen, the coupling efficiency is as high as $50-90 \%$. Linear dimensions and terminations applied allow for pulses up to a width of $12 \mathrm{~ns}$ and up to a voltage level of $4-5 \mathrm{kV}$ on the outside conductor. These pulse parameters proved to be sufficient to upset the DUTs tested so far.

In more than 400 measurements a rectangular pulse of increasing voltage level was applied to different types of CMOS and TTL until the individual DUT was damaged. As well the pulse width $(3,6$ or $12 \mathrm{~ns})$ and its polarity were varied in single-shot or repetitive-shot experiments (500 shots per voltage at a repetition rate of $3 \mathrm{~Hz}$ ). The state of the DUT was continuously monitored by measuring both the current of the DUT circuit and that of the oscillator providing the operating signal for the DUT.

The results show a very good reproducibility within a set of identical samples, remarkable differences between manufacturers and lower thresholds for repetitive testing, which indicates a memory effect of the DUT to exist for voltage levels significantly below the single-shot threshold.

\section{Introduction}

Object of this paper is the performance of electronic components when interfered by HF-signals. It is the goal of the measurements to collect data on the vulnerability of components exposed to HPM- or UWB-signals beyond some $100 \mathrm{MHz}$. The signal imposed on the operating signal of the DUT was varied with respect to voltage level, pulse width, repetition rate and polarity of the pulse to get information about the dependence of upset or damage on these parameters. Understanding these effects will help to better design protection measures of components and subsystems against unintentional and intentional electromagnetic threats.

\section{Measurement setup}

The operating signal to control the performance of the DUT is generated by a 7400-type CMOS, delivering a steady oscillation to the DUT (Fig. 1). As well the oscillator current I_Osci and I_DUT are monitored during the measurements. The HF-generator feeds the shield of the coaxial system on the DUT-side and couples into the signal line.

Due to the length of the coupling system $(1 \mathrm{~m})$ a pulse of $12 \mathrm{~ns}$ is the maximum width to prevent the end of the coupled pulse from interfering with its reflection from the other end of the line. The coupling efficiency strongly depends on the input impedance of the DUT. Especially during the process of upset with increasing voltage level this impedance varies almost unpredictably, depending on the actual state and failure mechanism of the DUT. To test the coupling efficiency, the DUT was replaced by a fixed impedance of 10, 100, 470 and $1000 \mathrm{Ohm}$, and the transfer function of coupling was calculated from the Fourier transformation of the primary and secondary (response) voltage signals. Figure 2 shows an efficiency of approximately $65 \%$ for the amplitudes in the time domain at a termination of $100 \mathrm{Ohm}$. 


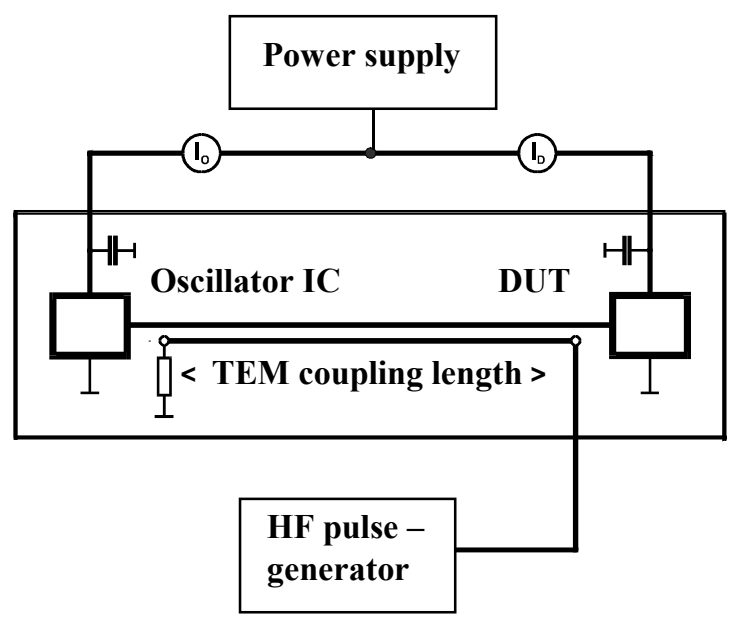

Fig. 1. Measurement setup.

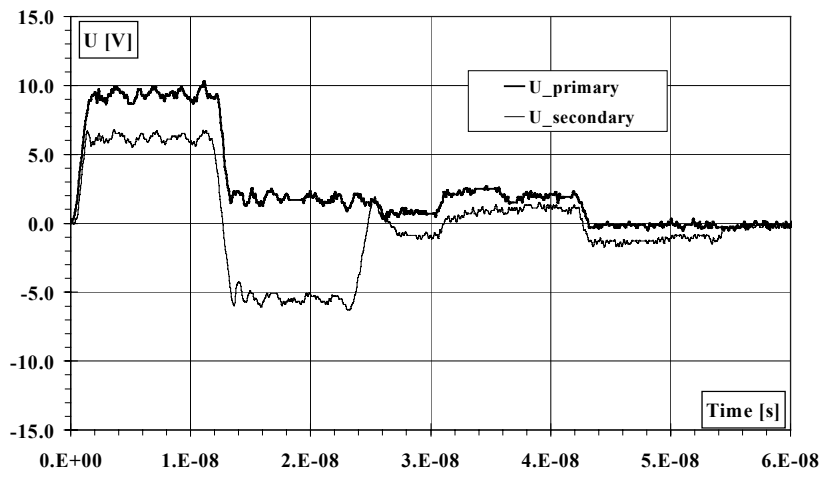

Fig. 2. Coupling for R_term $=100 \mathrm{Ohm}$.

The parallel pattern of spectral density of both signals (Fig. 3, right ordinate) shows the coupling mechanism to be almost independent from frequency, the transfer function $\mathrm{TF}$ $=$ U_sec/U_prim is oscillating around a constant level close to 1 (Fig. 3, left ordinate).

These calibration measurements were performed with two different widths of the test signal ( $6 \mathrm{~ns}$ and $12 \mathrm{~ns}$ ) and for both polarizations to get four individual TFs for each terminating impedance. The results show a very good independence from the pulse shape and thus, proved the linearity of the coupling system, making the threshold measurements comparable for all pulse shapes used.

As a final result of the calibration procedure, Fig. 4 shows the calculated response of the signal line due to a unipolar normalized synthetic input signal for different terminations. The response voltage increases with increasing impedance up to almost $100 \%$ for an open circuit.

\section{Measurements}

Out of 9 different types of TTLs and 13 types of CMOS, covering the range from "standard", "advanced" to "fast", more

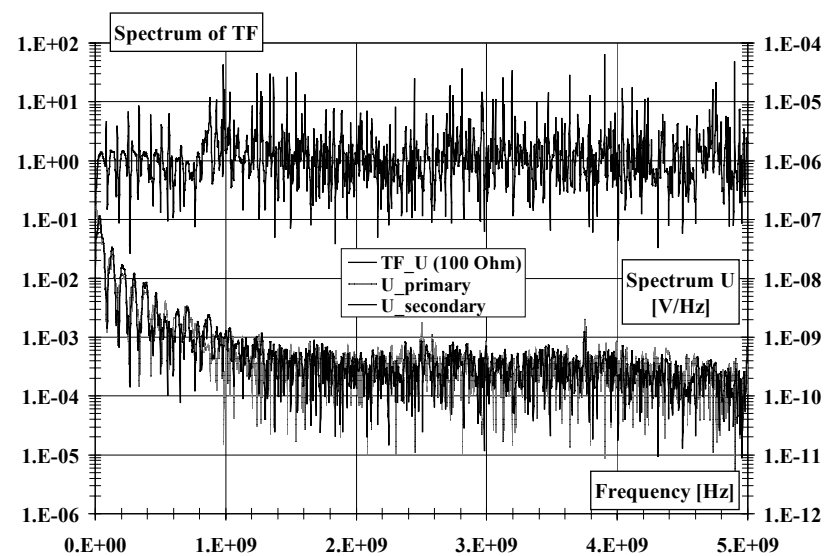

Fig. 3. Spectral density of voltage signals (right ordinate) and transfer function of coupling (left). Test pulse: $12 \mathrm{~ns}$ width, pos. polarity, R_term $=100$ Ohm.

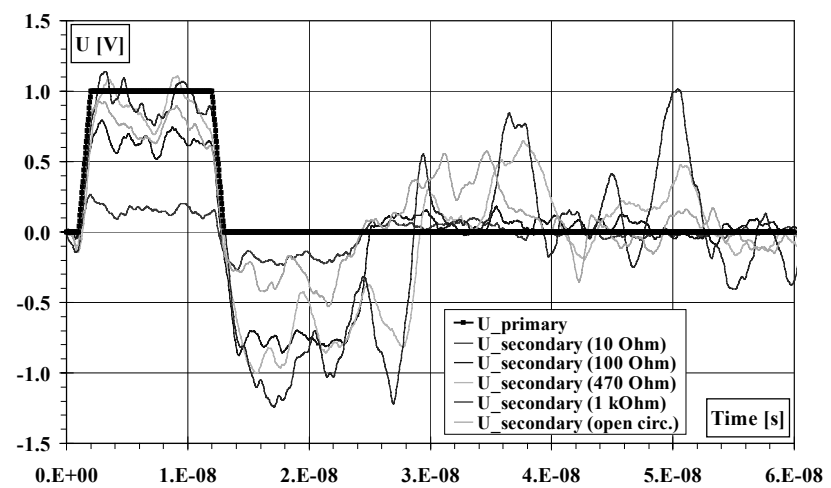

Fig. 4. Coupling efficiency from shield to signal line for an analytical input signal: Response U_secondary calculated from measured TFs for different terminations.

than 400 individual components were tested until burnout. These approximately 20 samples/type were used to vary the pulse width in 3 steps ( 3,6 and $12 \mathrm{~ns})$, the polarity (pos./neg.), the repetition parameter (single shot or 500 shots per voltage level) and to get statistical information.

For an individual measurement the primary voltage was gradually increased by voltage steps of $200 \mathrm{~V}$ each starting from zero, and one or 500 shots were applied. With increasing voltage a change of the current I_Osci and/or I_DUT indicated the beginning of failure mechanisms within the DUT, and the process was continued until burnout or latch up. As an example, Fig. 5 shows these currents vs. primary voltage for all the experiments done so far with the TTL SN7400N. Triangles indicate single shot experiments and square symbols stand for repetitive series. The size of the symbol is a measure of the pulse width: $12 \mathrm{~ns}$ (large), $6 \mathrm{~ns}$ (medium size) and $3 \mathrm{~ns}$ (small symbol).

Three features are obvious:

1. A general dependence on the type of signal

2. Increasing thresholds with decreasing pulse width 


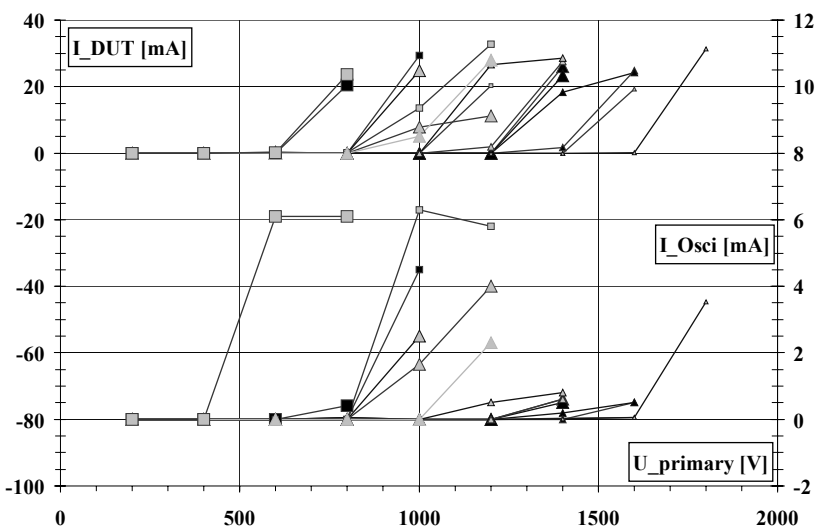

Fig. 5. Coupling of HF-signals into TTL, type SN7400N. Effect of increasing voltage, pulse width and repetition on I_DUT (left ord.) and I_Osci (right).



Fig. 6. Coupling of HF-signals into CMOS: threshold voltage for burnout. Pulse width 3, 6 and 12 ns, pos. polarity. Triangle: single shot, square: multiple shots (500/voltage level). Light colors: Latch up.

3. For a given pulse width: lower thresholds for multiple shots compared to single shots

These statements principally hold for all components tested.

\section{Results and discussion}

In Fig. 6 (CMOS) and Fig. 7 (TTL) all results for positive polarity are condensed to give a survey of common effects and differences. Single shot experiments (triangles) generally cause the thresholds for burnout to be higher than those for repetitive series (squares) and, within a type of symbol and within the error margin of about $200 \mathrm{~V}$, the thresholds increase with decreasing pulse width. Repeating the identical procedure with different samples of the same type of DUT normally showed a very good coincidence, failures occurred at the identical voltage level, but there were exceptions: Some individual CMOS components - of the $74 \mathrm{HC} 00 \mathrm{~N}-, 74 \mathrm{HCT} 00 \mathrm{~N}$-family and the lower four types in Fig. 6 - showed latch up instead of burnout. After the cou-

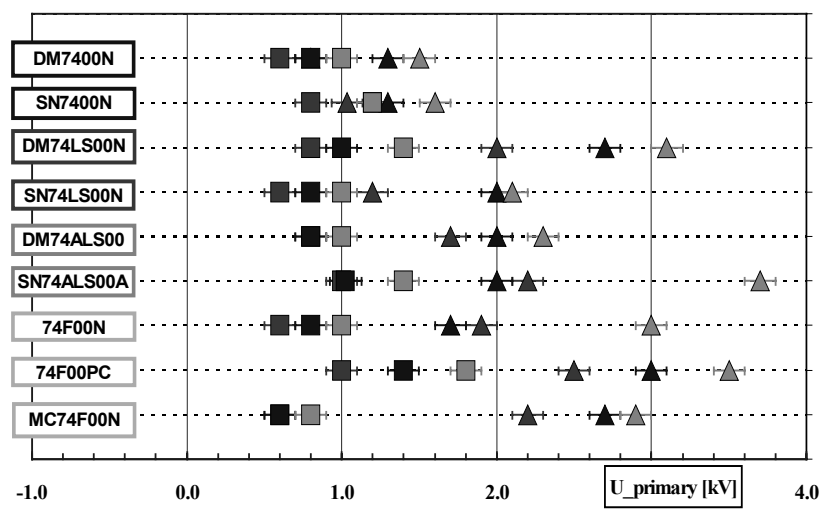

Fig. 7. Coupling of HF-signals into TTL: threshold voltage for burnout. Pulse width 3, 6 and 12, pos. polarity. Triangle: single shot, square: multiple shots (500/voltage level).

pling experiment all four gates of the DUT were tested, and the status of (entrance) gate 3 (still operating/damaged?) was taken as additional parameter to describe the test result. Different results and different patterns of the currents strongly indicate different damaging mechanisms to occur not only between the different types of DUTs but in some cases within a family itself. Studying these effects joined by microscopic investigations of the layout to spot defects is still under work.

To generalize the first results all components failed within a primary voltage range of $1-5 \mathrm{kV}$. But this relatively high level must be reduced by about $50 \%$ for the voltage on the signal line leading to a threshold of $500 \mathrm{~V}$ or less, if the pulse is repeated.

Still lower are the thresholds of upset, the voltage level at which the currents change by a margin at which proper functioning of the DUT can no longer be expected.

First interpretations applying an empirical "aging"-model with a lower voltage limit Uo depending on pulse width every shot above this limit contributes to the later damage at a higher voltage - are very promising. As a next step additional measurements using smaller pulses such as UWB and higher repetition numbers are planned to test and verify this model.

Acknowledgements. This work is supported by the German Ministry of Defence. 